\title{
Virus-induced gene silencing for phenylalanine ammonia-lyase affects pepper adaption to low temperature
}

\author{
G.-X. CHENG ${ }^{1}$, J.-T. SUN ${ }^{1}$, J.-P. SHANG ${ }^{2}$, and Z.-H. GONG ${ }^{1 *}$ \\ College of Horticulture, Northwest A\&F University, Yangling, Shaanxi 712100, P.R. China ${ }^{1}$ \\ Tianjin Vegetable Research Center, Tianjin 300192, P.R. China ${ }^{2}$
}

\begin{abstract}
Reverse genetics approaches in plants rely on post-transcriptional gene silencing to study the function of genes. In particular, virus-induced gene silencing (VIGS) has been successfully applied to identify gene function in some crops. To date, it is unclear whether phenylalanine ammonia-lyase (PAL) is involved in low temperature tolerance in the pepper. Here, we used an agroinfiltration protocol with tobacco rattle virus (TRV) constructs containing partial sequences from CaPAL for VIGS to test its role in anthocyanin biosynthesis and response to low temperature in the pepper (Capsicum annuum). We found that accumulation of anthocyanins in the leaves of pepper plants transformed with the TRV2:CaPAL vector was significantly reduced compared with peppers transformed with the empty TRV2 vector (TRV2:00). A significant reduction in expression of genes related to anthocyanins synthesis was also detected in peppers transformed with TRV2:CaPAL. When silenced pepper plants were exposed to a low temperature, we found decreased antioxidant system, PAL activity, and photosynthesis in plants transformed with TRV2:CaPAL compared with peppers transformed with TRV2:00. Low transcriptions of cold stress-response genes demonstrated that pepper tolerance to low temperature decreased. Future studies focused on the interaction between $C a P A L$ and other abiotic and biotic stressors will shed further light into the role of $C a P A L$ in stress response.
\end{abstract}

Additional key words: anthocyanins, Capsicum annuum, photosynthesis, transgenic plants, TR2:CaPAL vector.

\section{Introduction}

A major challenge for biology is to integrate research approaches to address how organisms adapt to environmental stress (Conde et al. 2011). To achieve this, understanding the function of genes is indispensable. To date, most reverse genetics approaches in plants have relied on post-transcriptional gene silencing to study the function of genes (Zhai et al. 2017). This is especially suitable for non-model species (Tavakol 2018), which do not have a catalogue of well-characterized mutants, but it is also a useful tool for model species (Jupin 2013). Virus-induced gene silencing (VIGS) suppresses gene transcripts, and has been successfully used to characterize the function of plant genes in Glycine $\max$ (Jeong et al. 2005). The most widely used VIGS vectors are based on the tobacco rattle virus (TRV), and TRV-mediated VIGS is used to silence genes in a number of crops, including rice (Purkayastha et al. 2010) and tomato (Fantini and Giuliano 2016).

Anthocyanins are plant pigments derived from the phenylpropanoid pathway (Van Tunen and Mol 1991, Oren-Shamir 2009), and the anthocyanin biosynthetic pathway is an important branch of flavonoid pathway (Ubi 2007). The content and stability of anthocyanins are affected by environmental factors (Jenshi Roobha et al. 2011, Kovačević et al. 2015). In general, plants can have higher tolerance for abiotic or biotic stressors by accumulating anthocyanins (Filiz et al. 2010, Schulz et al. 2015).

Phenylalanine ammonia-lyase, encoded by the $P A L$ gene, is the first key enzyme involved in the phenylpropanoid pathway, and it is important for anthocyanin biosynthesis, and thus plant defence against environment stresses (Pombo et al. 2011, Aza-González et al. 2013, Zhu et al. 2015). The PAL has been successfully isolated from rice and pepper (Kim and Hwang 2014,

Submitted 7 June 2018, last revision 7 March 2019, accepted 26 March 2019.

Abbreviations: APX - ascorbate peroxidase; $\mathrm{c}_{\mathrm{i}}$ - intercellular $\mathrm{CO}_{2}$ concentration; $\mathrm{g}_{\mathrm{s}}$ - stomatal conductance; MDA - malondialdehyde; PBS - phosphate buffer solution; PAL - phenylalanine ammonia-lyase; $\mathrm{P}_{\mathrm{N}}$ - net photosynthetic rate; POD - peroxidase; qPCR - quantitative PCR; ROS - reactive oxygen species; SOD - superoxide dismutase; E - transpiration rate; TRV - tobacco rattle virus; TRV2:00 - empty TRV vector; TRV2:CaPAL - TRV2 vector with CaPAL gene; TRV2:CaPDS - TRV2 vector with CaPDS gene; VIGS - virus-induced gene silencing.

Acknowledgements: This work was supported by the National Key R\&D Program of China (No. 2016YFD0101900) and the National Natural Science Foundation of China (No.31772309).

* Corresponding author; e-mail: zhgong@nwsuaf.edu.cn 
Tonnessen et al. 2014). Environmental conditions can regulate transcription of $P A L$ gene and activate respective protein function (Christopoulos and Tsantili 2015). Phenylalanine ammonia-lyase knock-out plants show damage induced by environmental stresses (Rohde 2004).

Pepper is cultivated widely throughout the world (Pimenta et al. 2016). However, cold weather often results in significant reduction of its yield and quality. Although PAL (GenBank: KF279696) in the pepper is a ratelimiting enzyme in the plant defence system, studies on its biological function have been restricted to its role in anthocyanins biosynthesis. The pall knockout plants are deficient in anthocyanin pigments suggesting that PAL1 has an important role in flavonoid biosynthesis (Huang et al. 2010). Here, we employed VIGS to knock down the $P A L$ gene in a color-leafed pepper cultivar $\mathrm{Z} 1$ to investigate the effect of $P A L$ expression on anthocyanins biosynthesis and response to low temperature. Our work will provide insights into the mechanism of pepper anthocyanin biosynthesis and offer a potential for improving breeding in the future.

\section{Materials and methods}

Plants and cultivation: The pepper (Capsicum annuum L.) purple-leafed cultivar Z1 was used for all experiments (Fig. 1 Suppl.). Pre-germinated seeds were sown in $0.2-\mathrm{dm}^{3}$ nutritional bowls with $85 \mathrm{~g}$ of matrix and germinated at a temperature of $28{ }^{\circ} \mathrm{C}$. Seedlings at the cotyledon stage were transferred to growth cabinets with a 16-h photoperiod, an irradiance of $500 \mu \mathrm{mol} \mathrm{m} \mathrm{m} \mathrm{s}^{-1}$, day/ night temperatures of $22 / 18^{\circ} \mathrm{C}$, and a relative humidity of $75-80 \%$.

Construction of TRV plasmids and generation of $\boldsymbol{C a P A L}$-silenced plants: The tobacco rattle virus (TRV)based VIGS system was used to silence the CaPAL gene in the Z1 pepper cultivar. The empty vector (TRV2:00) was used as a negative control. Following previous studies, the CaPDS gene (GenBank: X68058.1), encoding a phytoene desaturase in biosynthesis pathway of carotenoids, was used as a positive visual marker, whose silencing produces a photobleaching phenotype in plant newly-growing tissues/ organs (Cunningham and Gantt 1998). A fragment of the CaPDS coding region was amplified using gene-specific primers forward VCaPDS with an EcoRI restriction site, and reverse VCaPDS with a BamHI restriction site (Table 1 Suppl.). The resulting product was inserted into the TRV2 vector to generate the TRV2:CaPDS vector as a positive control (Fig. 2 Suppl.).

The full length of CaPAL mRNA sequence (GenBank: KF279696) contains 2318 base pairs (bp) with an open reading frame, and a 488 bp fragment (location: 1179 $2266 \mathrm{bp}$ ) from the 3 ' end of the gene was cloned in the TRV2 vector using gene-specific primers and generated the TRV2:CaPAL (Table 1 Suppl., Fig. 2 Suppl.). To ensure the specificity of CaPAL gene and avoid a product of other homologous genes, the primer should be designed in the nonconservative domain of the gene.
The TRV1, TRV2:00 (negative control), TRV2:CaPDS, and TRV2:CaPAL vectors were transformed into the Agrobacterium tumefaciens strain GV3101. The GV3101 cells carrying TRV1 were mixed with TRV2:00, TRV2:CaPDS, and TRV2:CaPAL at a $1: 1: 1$ ratio. The suspensions of Agrobacterium inoculation containing TRV1, TRV2:00, TRV2:CaPDS, and TRV2:CaPAL (with absorbance at $600 \mathrm{~nm}$ equal to 1.0 ) were infiltrated into the fully expanded cotyledons of wild type pepper using a sterilized syringe without the needle. The Agrobacteriuminoculated pepper plants were grown in a growth chamber at $18{ }^{\circ} \mathrm{C}$ and a $45 \%$ relative humidity in darkness for $2 \mathrm{~d}$, and then transferred into a growth chamber at $22^{\circ} \mathrm{C}$, a $60 \%$ relative humidity, a $16-\mathrm{h}$ photoperiod, and an irradiance of $500 \mu \mathrm{mol} \mathrm{m} \mathrm{m}^{-2} \mathrm{~s}^{-1}$. When the photobleaching symptom appeared on the leaves of pepper seedlings inoculated with TRV2:CaPDS (representing the success of VIGS), the young leaves of TRV2:CaPAL and TRV2:00 were sampled for testing silencing efficiency and stress treatments.

The selected TRV2:CaPAL and TRV2:00 plants were placed at $4{ }^{\circ} \mathrm{C}$ for $16 \mathrm{~h}$ for low temperature treatment. Young leaves were sampled for measuring metabolites as described below. Three biological replicates were conducted for each treatment, and each replicate contained three pepper seedlings.

Measurement of anthocyanin content and PAL activity: Anthocyanin content was quantified following methods of Christie et al. (1994) with slight modifications. After one month of injection, fresh leaves $(1.0 \mathrm{~g})$ were grinded in liquid nitrogen, dissolved with a homogenization solution $\mathrm{HCl}$ (conc.) : methanol (100\%) 1:100, v/v, and incubated at $4{ }^{\circ} \mathrm{C}$ for $4 \mathrm{~h}$. The mixture was centrifuged at $24170 \mathrm{~g}$ for $20 \mathrm{~min}$ and the supernatant was measured spectrophotometrically at $530 \mathrm{~nm}$.

Activity of PAL was determined by the production of cinnamate at $30^{\circ} \mathrm{C}$ during $1 \mathrm{~h}$, as measured by the change in absorbance at $290 \mathrm{~nm}$ following published protocol (Zhou et al. 1990) with slight modifications. After one month of injection, fresh leaves $(1.0 \mathrm{~g})$ were grinded in liquid nitrogen and dissolved in $0.1 \mathrm{M}$ sodium borate buffer (pH 8.8) containing $5 \mathrm{mM}$ mercaptoethanol and $5 \%(\mathrm{~m} / \mathrm{v})$ tetrapyrrolidone. A $0.1 \mathrm{~cm}^{3}$ of supernatant was added to an assay mixture with $0.02 \mathrm{M}$ phenylalaninein to a total volume of $3.1 \mathrm{~cm}^{3}$ after the buffer was centrifuged $(2832 \mathrm{~g}$ ) for $15 \mathrm{~min}$. Assays were performed in triplicate. Under the experimental conditions, an increase in absorbance at $290 \mathrm{~nm}$ was linear for up to $1 \mathrm{~h}$. A unit (U) of PAL was defined as a $1 \%$ loss in PAL activity per min.

Extraction of RNA and real time quantitative PCR: Quantitative PCR (qPCR) was conducted to test the silencing efficiency after the positive control showed symptoms of photobleaching. Total RNA was isolated following the method of Guo et al. (2012), and cDNA was synthesized using a PrimeScript kit (Takara, Dalian, China) according to the manufacturer's instructions. Primers were designed to generate 150 - 250 base pair fragments using the Primer 5 software, and are listed in 
Table 1 Suppl. The PCR and detection were performed as described previously (Feng et al. 2012). The $2^{-\Delta \Delta \mathrm{Ct}}$ method was used to analyze the relative gene expressions (means from three replicates). The qPCR was performed using $i Q 5$ (Bio-Rad, Hercules, USA), and the ubiquitin-conjugating gene $\mathrm{CaUbi3}$ (AY486137) was used as a reference gene.

Photosynthetic parameters measurement: Photosynthetic rate $\left(\mathrm{P}_{\mathrm{N}}\right)$, stomatal conductance $\left(\mathrm{g}_{\mathrm{s}}\right)$, intercellular $\mathrm{CO}_{2}$ concentration $\left(\mathrm{c}_{\mathrm{i}}\right)$ and transpiration rate $(\mathrm{E})$ were determined by a portable photosynthesis system (LI-6400, LI-COR, Lincoln, NE, USA) mounted with a $\mathrm{red} / \mathrm{blue}$ LED diode (6400-02B, LI-COR) on the youngest fully developed leaves (the $4^{\text {th }}$ leaf from the top). All measurements were conducted at $22 \pm 2{ }^{\circ} \mathrm{C}$ and an ambient humidity of $75 \pm 5 \%$. Photosynthetic photon flux density at the leaf surface was set at $500 \mu \mathrm{mol} \mathrm{m} \mathrm{m}^{-1}$.

Measurement of antioxidant enzymes and malondialdehyde content: To measure superoxide dismutase (SOD) activity, $0.5 \mathrm{~g}$ of fresh samples were grinded in a mortar and pestle with $5 \mathrm{~cm}^{3}$ of phosphate buffer solution (PBS) containing $50 \mathrm{mM}$ PBS, $25 \mathrm{mM}$ nitrotetrazolium blue chloride, $0.003 \mathrm{mM}$ riboflavin, and $0.1 \mathrm{mM}$ ethylene diamine tetraacetic acid at $\mathrm{pH} 7.8$. Homogenates were centrifuged at $24170 \mathrm{~g}$ and $4{ }^{\circ} \mathrm{C}$ for $15 \mathrm{~min}$. The supernatants were exposed to an irradiance of $500 \mu \mathrm{mol} \mathrm{m}{ }^{-2} \mathrm{~s}^{-1}$ for $20 \mathrm{~min}$.

Activity of SOD was quantified spectrophotometrically at $560 \mathrm{~nm}\left(\mathrm{~A}_{560}\right.$ of the control containing water instead of the supernatant was determined in darkness). The SOD activity was calculated based on Dionisio-Sese and Tobita (1998). One unit (U) of SOD activity was defned as the amount of the enzyme which caused a $50 \%$ inhibition of initial rate of the reaction in the absence of the enzyme.

To measure peroxidase (POD) activity, $0.1 \mathrm{~g}$ of fresh samples were grinded in a mortar and pestle with $5 \mathrm{~cm}^{3}$ of PBS (20 mM, pH 6.0). Homogenates were centrifuged at $24170 \mathrm{~g}$ and $4{ }^{\circ} \mathrm{C}$ for $10 \mathrm{~min}$. The supernatants were exposed to an irradiance of $500 \mu \mathrm{mol} \mathrm{m} \mathrm{m}^{-2} \mathrm{~s}^{-1}$ for $20 \mathrm{~min}$. Activity of POD was quantified spectrophotometrically at $470 \mathrm{~nm}$, and absorbances were recorded every $30 \mathrm{~s}$. The POD activity was calculated by the method of DionisioSese and Tobita (1998). One unit (U) of POD activity was defined as a change in absorbance per minute.

For ascorbate peroxidase (APX) activity, $1.0 \mathrm{~g}$ of samples were frozen in liquid nitrogen, ground using a mortar and pestle, and immediately homogenized with $50 \mathrm{mM}$ 4-(2-hydroxyethyl)-1-piperazineethanesulfonic acid buffer solution ( $\mathrm{pH} 7.2$ ) containing $1.0 \mathrm{mM}$ $\mathrm{Na}_{2}$ EDTA, $1 \mathrm{mM}$ sodium ascorbate, $3 \%(\mathrm{~m} / \mathrm{v})$ insoluble polyvinylpyrrolidone, and $0.3 \%(\mathrm{v} / \mathrm{v})$ polyethylene glycol tert-octylphenyl ether at $4{ }^{\circ} \mathrm{C}$ for $15 \mathrm{~min}$. The homogenates wwere filtered through nylon cloth and centrifuged at $24710 \mathrm{~g}$ for $10 \mathrm{~min}$. Ascorbate peroxidase activity was determined following the method from Nakano and Asada (1987). One unit (U) of APX activity was defined as a decrease of absorbance at $290 \mathrm{~nm}$ per minute.

To measure malondialdehyde (MDA) content, $0.2 \mathrm{~g}$ of samples were ground in liquid nitrogen using a pestle and mortar into which $5 \mathrm{~cm}^{3}$ of ice-cold $10 \%(\mathrm{~m} / \mathrm{v})$ trichloroacetic acid was added. Content of MDA was measured following Dionisio-Sese and Tobita (1998) with modifications. Briefly, the homogenates were centrifuged at $24170 \mathrm{~g}$ for $15 \mathrm{~min}$ and the supernatants were added to the same volume of a $10 \%(\mathrm{~m} / \mathrm{v})$ thiobarbituric acid solution containing $0.6 \%(\mathrm{~m} / \mathrm{v})$ trichloroacetic acid. The mixtures were heated at $100{ }^{\circ} \mathrm{C}$ for $20 \mathrm{~min}$ and the reaction was rapidly halted by placing the mixtures into an ice bath. The cooled reaction solutions were then centrifuged at $24170 \mathrm{~g}$ for $10 \mathrm{~min}$, and the absorbance of the supernatants was measured at 450, 532, and $600 \mathrm{~nm}$.

Data analysis: The experiment was designed to be completely randomized within the growth chamber with three replicates per treatment, and the entire experiment was performed in duplicate. The data were analyzed using one-way analysis of variance using the SPSS 11.0 software. Statistical significance was inferred at $P<0.05$. The data were presented as means \pm SDs of the three replicates for all measured parameters.

\section{Results}

To understand the role of CaPAL in the pepper tolerance to low temperature, we generated plants to knock down its expression through VIGS. Agrobacterium stains harboring TRV2:00, TRV2:CaPAL, and TRV2:CaPDS were injected to the cotyledons of purple-leafed pepper line Z1 (Fig. 1 Suppl.). A month after injection, plants treated with the positive control, TRV2:CaPDS, showed photobleaching, and bleaching the new leaves was maintained until the seedlings died. Furthermore, CaPDS-silenced peppers grew at a slower rate compared to peppers treated with the empty vector TRV2:00 (the negative control). We did not observe obvious symptoms on pepper seedlings treated with TRV2:00 (Fig. 1 $A, B$ ). Plants transformed with TRV2:CaPAL showed changes in growth and color compared to the peppers of TRV2:00. The CaPAL-silenced leaves were completely green (Fig. 1C). Interestingly, peppers transformed with TRV2:CaPAL had a significantly lower anthocyanin content and PAL activity compared to those transformed with TRV2:00 (Fig. 1D-F). These data support that VIGS successfully knocked down the expression of the target gene in pepper, and that CaPAL was silenced with a high efficiency one month after injection (Fig. 1E).

To understand the role of $C a P A L$ in pepper tolerance to low temperature, $C a P A L$-silenced peppers were placed at $4{ }^{\circ} \mathrm{C}$ for $16 \mathrm{~h}$, and plants transformed with TRV2:CaPAL showed more wilting compared to the negative control plants transformed with TRV2:00 (Fig. 2A). Comparing new leaves of the $C a P A L$-silenced peppers and the control, we found that the apexes and sides of the new leaves in the $C a P A L$-silenced seedlings were shrunk compared to those transformed with TRV2:00 under low temperature conditions.

We next investigated the effects of low temperature on $C a P A L$-silenced plants at the physiological level. Low 
temperature treatment increased the total anthocyanins content in both silenced peppers and the negative control; however, the negative control plants (TRV2:00) had higher anthocyanins content than the $C a P A L$-silenced plants (Fig. $2 B$ ). Under normal conditions, PAL activity in plants transformed with TRV2:CaPAL was lower compared to peppers of TRV2:00. Low temperatures led to a higher PAL activity in the negative controls transformed with TRV2:00 but to a lower PAL activity in TRV2:CaPAL plants (Fig 2C). Content of MDA increased in the leaves of TRV2:00 and TRV2:CaPAL under low temperature, and the rate of increase was higher in the $C a P A L$-silenced plants (Fig. 3A). Interestingly, although peppers exposed to low temperature had higher anthocyanins content, anthocyanin amounts in CaPAL-silenced peppers were lower than those in peppers of TRV2:00 (Fig. 2) suggesting that silencing CaPAL led to a reduced anthocyanins syntheses and the accumulation of MDA in plants.

We tested the activities of antioxidant enzymes to examine whether CaPAL-silencing affected pepper tolerance to low temperature. Under normal conditions, enzyme activities were similar between silenced peppers and the negative control. Enzyme activities were higher in plants exposed to low temperature and the negative controls had significantly higher enzyme activities compared to the silenced peppers at low temperature conditions (Fig. 3B-D).

Furthermore, photosynthetic parameters decreased after low temperature treatment (Fig. 4). Under normal conditions, $\mathrm{P}_{\mathrm{N}}$, and $\mathrm{E}$ in the silenced peppers were higher than those in the negative control, and there was no difference in $g_{s}$ between the negative control and the silenced peppers. However, after low temperature treatment, all parameters were lower in the silenced peppers compared the negative control. Even E in the negative control was two-fold higher than in the silenced peppers. These results demonstrate that the silenced peppers had a poor photosynthesis capacity.

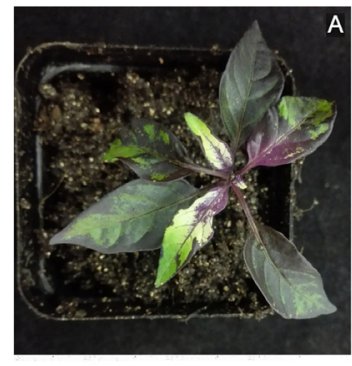

TRV2:CaPDS

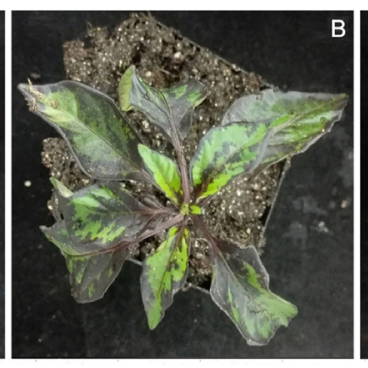

TRV2:00

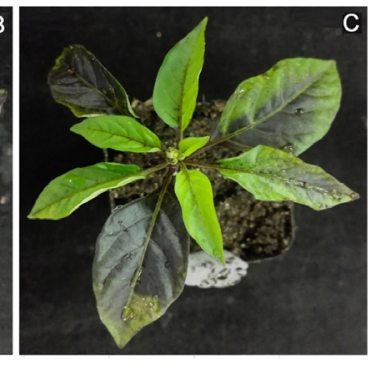

TRV2:CaPAL

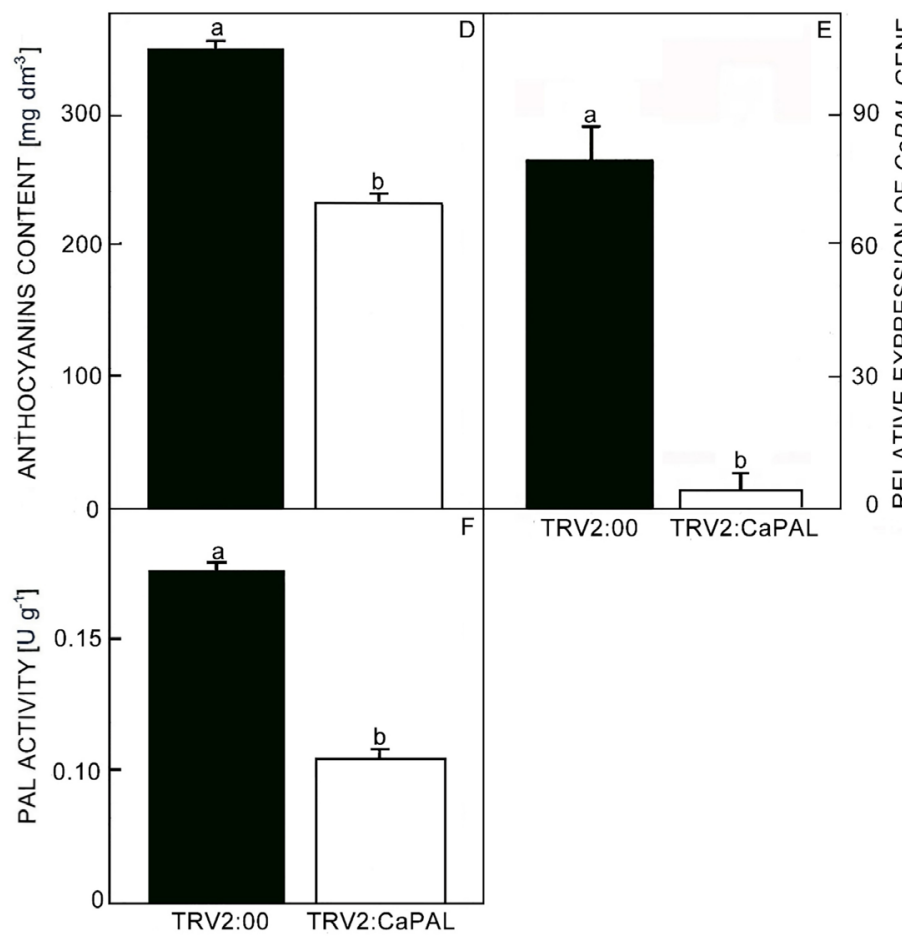

Fig. 1. Effect of $C a P A L$-silencing in pepper plants. Phenotypes of pepper seedlings transformed with TRV2:CaPDS as a positive control $(A)$, TRV2:CaPAL $(B)$, and the empty vector as a negative control $(C)$. Anthocyanins content $(D)$, expression of the $C a P A L$ gene $(E)$, and phenylalnine ammonia-lyase (PAL) activity $(F)$ in silenced pepper TRV2:CaPAL and negative control TRV2:00. The experiment was conducted with three biological replicates, and each replicate contained three pepper seedlings. Means \pm SDs, different lowercase letters mark significant differences at $P<0.05$. 
To further explore the effect of low temperature on CaPAL-silenced peppers, expressions of genes involved in pigment metabolism were investigated by real-time qPCR. Under normal conditions, expressions of anthocyaninsrelated genes in the negative control was higher than the silenced peppers (Fig. 5). After low temperature treatment, the $C a P A L$-silenced peppers did not show a difference in the expression of anthocyanins-related genes, whereas the TRV2:00 plants had higher expressions of these genes (Fig. 5A). For example, the TRV2:00 seedlings had higher expressions of CaMYC (a transcription factor from the bHLH_family), a transcription factor from the MYB family, UDP-glucose:flavonoid 3-glucosyltransferase, anthocyanin synthase, and chalcone synthase under low temperature, whereas the TRV2:CaPAL seedlings had a similar $C a M Y C$ expression at low temperature and at normal conditions. The expressions of carotenoids-related and chlorophyll-related genes were also different between the negative control and the $C a P A L$-silenced peppers, but expressions of all genes decreased under low temperature (Fig. 5B-C).

Expressions of antioxidant-related genes $C$-repeat binding factor 1, proline oxidoreductase 1 and superoxide dismutase were enhanced in both the silenced pepper TRV2:CaPAL seedlings and the control TRV2:00 seedlings, but much less in the former than in the latter (Fig. 6).

\section{Discussion}

Virus-induced gene silencing is considered to be an effective tool for analyzing gene function in plants. Over the last decade, VIGS has been used for both forward and reverse genetics to study gene function in various model plants (Ramegowda et al. 2014). Here, we demonstrate that VIGS could be effectively used to down-regulate a gene CaPAL involved in anthocyanin biosynthesis in peppers. The accumulation of anthocyanins allows plants to adapt to adverse environments (Landi et al. 2015), and PAL is an important enzyme in the anthocyanin biosynthetic pathway, catalyzing the non-oxidative deamination of phenylalanine to trans-cinnamate (Kim and Hwang 2014). Although the role of $P A L$ genes in stress response has been reported in Arabidopsis (Olsen et al. 2008), the effect of $P A L$ down-regulation on pepper anthocyanins has not been investigated. Hence, to test the involvement of PAL in anthocyanins biosynthesis in peppers, we took a VIGS approach to silence $P A L$ gene in pepper.

The PAL catalyzes the first step of the phenylpropanoid pathway and so the synthesis of diverse natural products based on the phenylpropane skeleton (Mauch-Mani and Slusarenko 1996). We characterized the role of CaPAL in pepper anthocyanins biosynthesis using plants with knocked down expression of CaPAL. We found that $C a P A L$ is a positive regulator of pepper anthocyanins

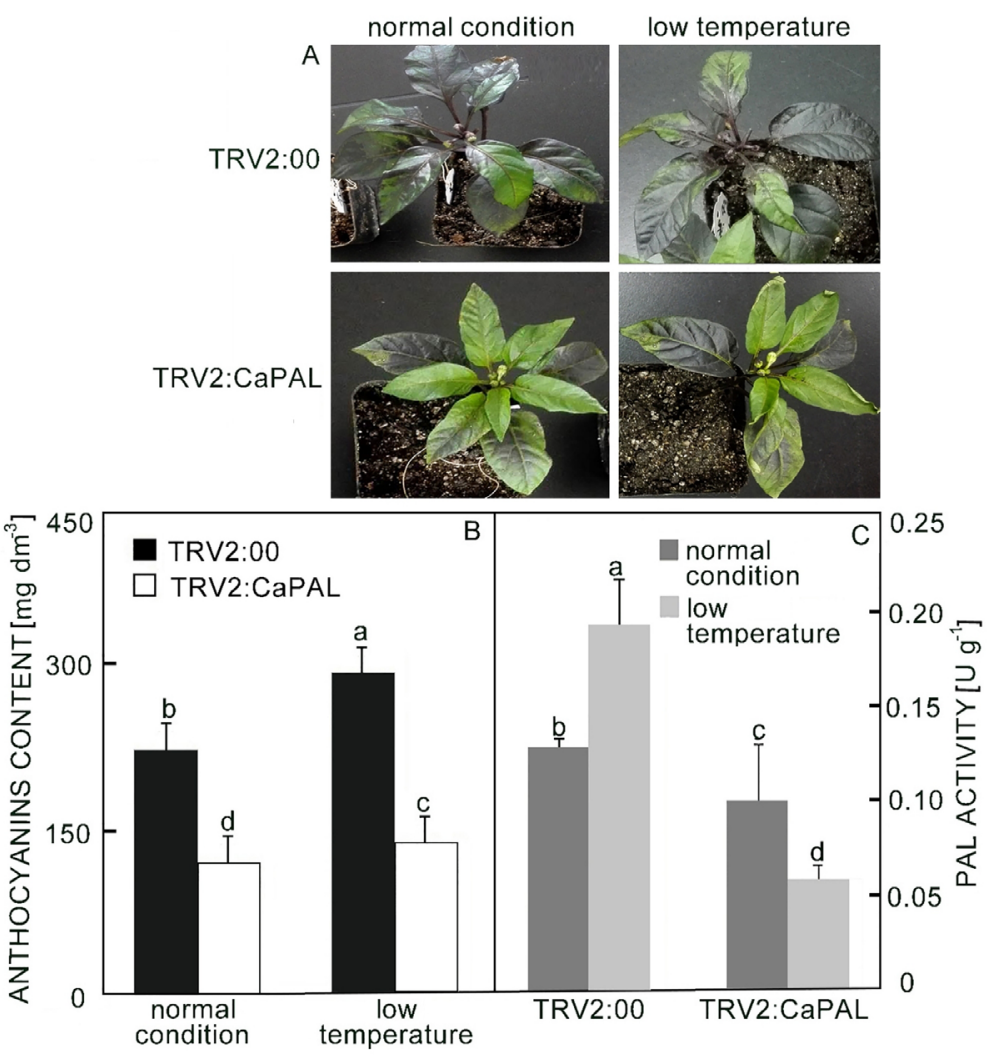

Fig. 2. Effect of low temperature $\left(4^{\circ} \mathrm{C}\right.$ for $\left.16 \mathrm{~h}\right)$ on phenotype and contributing parameters in silenced peppers. Phenotypes of silenced pepper TRV2:CaPAL and negative control TRV2:00 $(A)$; their anthocyanins content $(B)$; theirand PAL activity $(C)$. The experiment was conducted with three biological replicates, and each replicate contained three pepper seedlings. Means \pm SDs, different lowercase letters mark significant differences at $P<0.05$. 
biosynthesis, as knocking down CaPAL expression resulted in a significant decrease in anthocyanin content in pepper leaves. Silencing $P A L$ may have prohibited flux in the flavonoid pathway, thereby resulting in a low yield of some secondary metabolites (Olsen et al. 2008, Cheng et al. 2018). Expression of CaPAL significantly increases PAL activity, triggering synthesis of anthocyanins in plants
(Wang et al. 2000, Kim and Hwang 2014). The CaPAL positively regulates anthocyanin production in peppers (Cheng and Breen 1991). Furthermore, silencing CaPAL results in a reduced expression of anthocyanin-related genes (e.g., anthocyanin synthase and chalcone synthase), further suggesting that PAL is involved in the anthocyanin biosynthetic pathway.

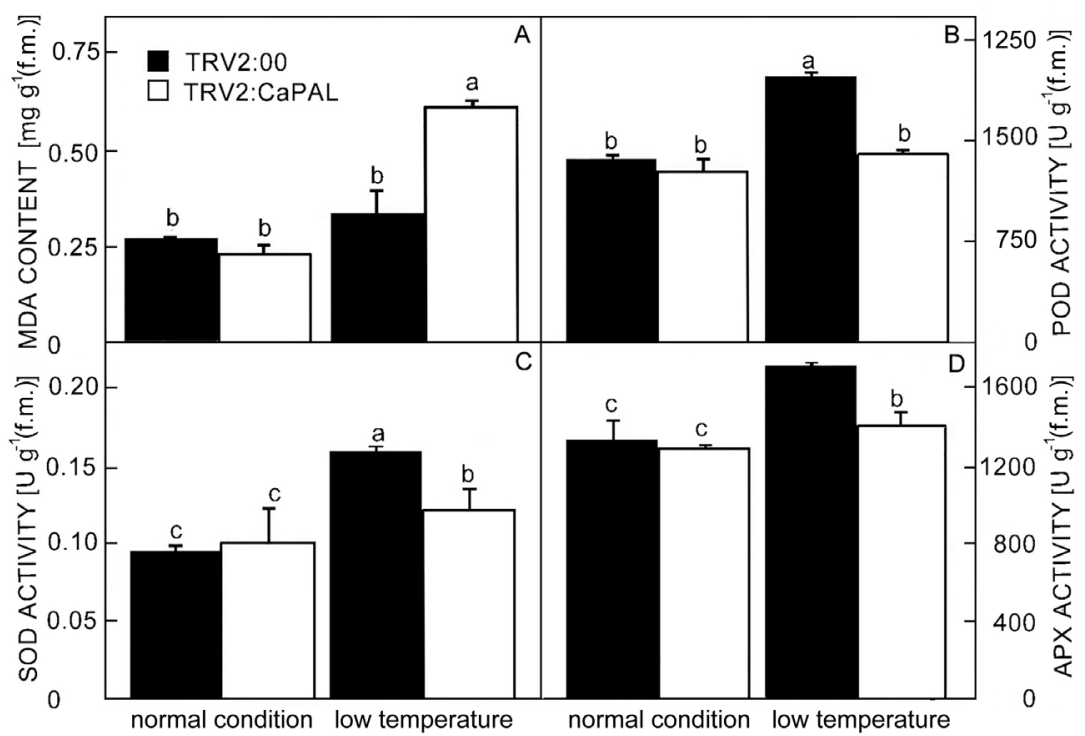

Fig. 3. Malondialdehyde (MDA) content and activities of antioxidant enzymes in peppers exposed to low temperature $\left(4^{\circ} \mathrm{C}\right.$ for $\left.16 \mathrm{~h}\right)$. $A$ - MDA content; $B$ - peroxidase (POD) activity; $C$ - superoxide dismutase (SOD) activity; $D$ - ascorbate peroxidase (APX) activity of silenced pepper TRV2:CaPAL and negative control TRV2:00. The experiment was conducted with three biological replicates, and each replicate contained three pepper seedlings. Means \pm SDs, different lowercase letters mark significant differences at $P<0.05$.

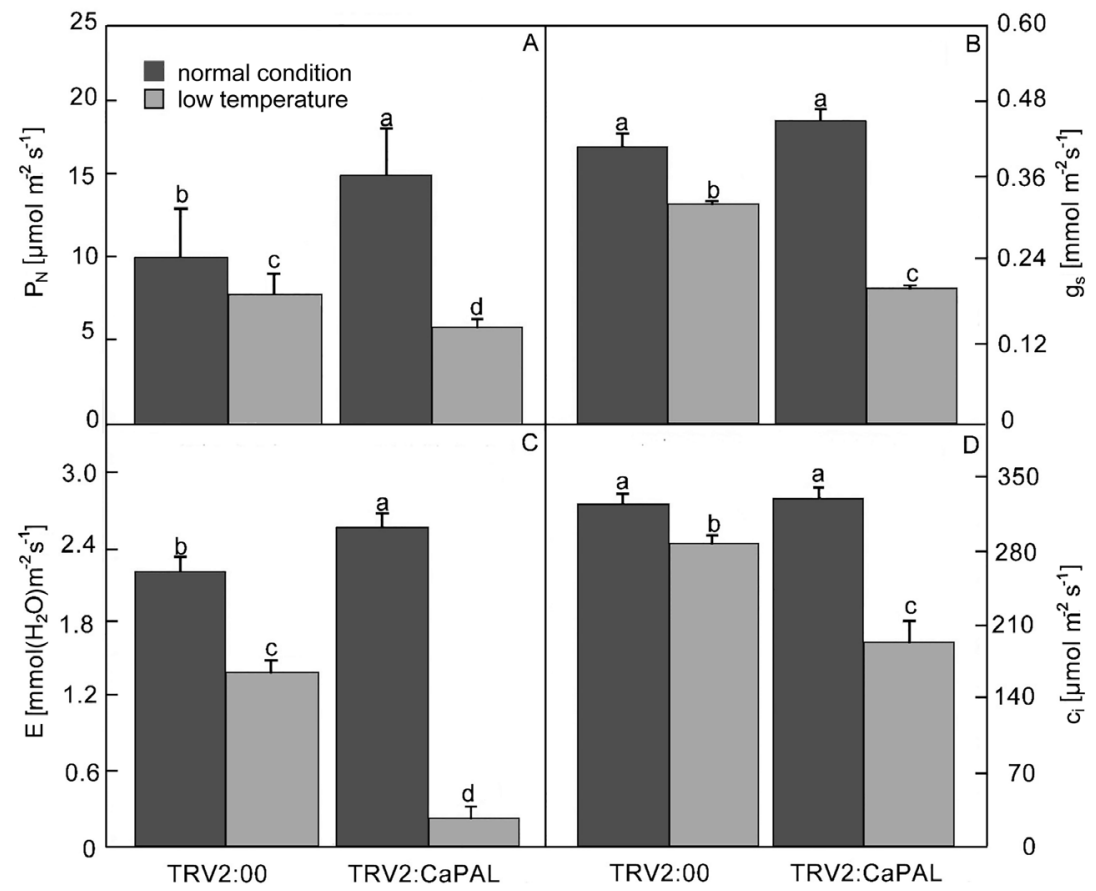

Fig. 4. Photosynthetic capacity in peppers exposed to low temperature $\left(4{ }^{\circ} \mathrm{C}\right.$ for $\left.16 \mathrm{~h}\right) . A$ - Photosynthetic rate $\left(\mathrm{P}_{\mathrm{N}}\right) ; B$ - stomatal conductance $\left(\mathrm{g}_{\mathrm{s}}\right) ; C$ - transpiration rate $(\mathrm{E}) ; D$ - intercellular carbon dioxide concentration $\left(\mathrm{c}_{\mathrm{i}}\right)$ of silenced pepper TRV2:CaPAL and negative control TRV2:00. The experiment was conducted with three biological replicates, and each replicate contained three pepper seedlings. Means $\pm \mathrm{SDs}_{2}$ different lowercase letters mark significant differences at $P<0.05$. 
Similar to the studies on PAL in Arabidopsis (Leyva et al. 1995), the knocking down CaPAL expression rendered pepper seedlings more sensitive to low temperature. Under low temperature, the pepper seedlings transformed with TRV2:CaPAL had a higher MDA content (Fig. 3) and lower activities of antioxidant enzymes compared to the negative control plants transformed with the empty vector TRV2:00. These results suggest that silencing CaPAL causes a reduced ability to remove reactive oxidative species (ROS), leading to an increased membrane damage.
Similarly, deficiency in anthocyanins resulting from knocking down genes involved in anthocyanin biosynthesis also made plants hypersensitive to ROS, resulting in the accumulation of insoluble protein aggregates under stressful conditions. Interestingly, our results are not in agreement with Kim and Hwang (2014), who reported that PAL overexpression can increase ROS burst and cell death. This may be explained by the fact that an optimal amount of ROS can improve plant growth under adverse conditions, whereas excess ROS are detrimental to plant
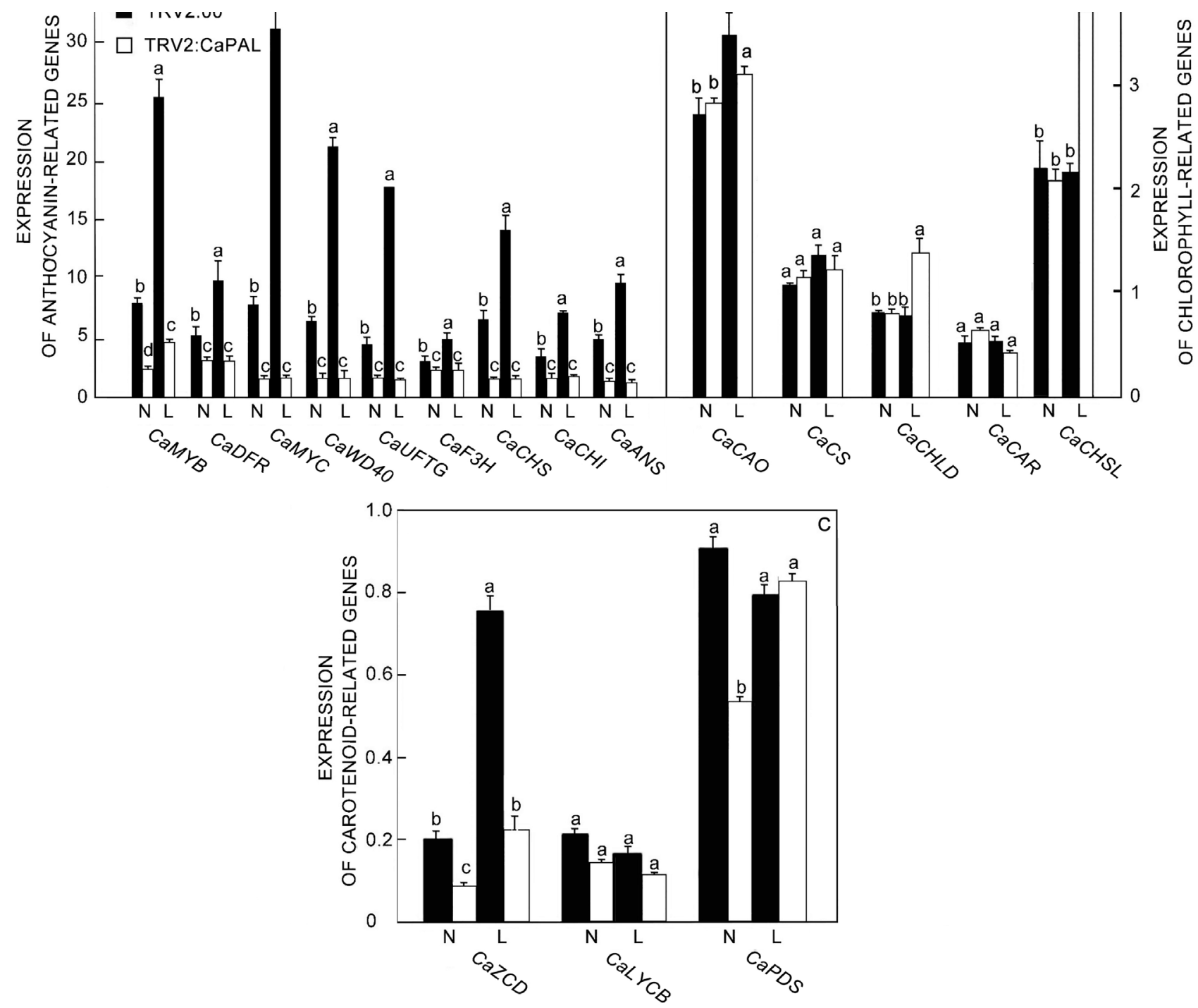

Fig. 5 Expressions of pigment-related genes in the leaves of silenced pepper TRV2-CaPAL and negative control plants TRV2:00 exposed to low temperature $\left(4^{\circ} \mathrm{C}\right.$ for $\left.16 \mathrm{~h}\right) . A$ - Expressions of anthocyanin-related genes a transcription factor from the MYB family $(C a M Y B)$, dihydroflavonol 4-reductase (CaDFR), a transcription factor from the bHLH family (CaMYC), a WD40repeat protein (CaWD40), UDP-glucose:flavonoid 3-O-glucosytransferase (CaUFGT), flavanone 3-hydroxylase (CaF3H), chalcone synthase (CaCHS), chalcone isomerase $(\mathrm{CaCH})$, anthocyanin synthase (CaANS); $B$ - expressions of chlorophyll-related genes chlorophyllide a osygenase (CaCAO), chlorophyll synthase (CaCS), D subunit of magnesium chelatase(CaCHLD), chlorophyll a reductase (CaCAR), a gene resulting in inhibition of chlorophyll synthesis (CaCHSL); $C$ - expressions of carotenoid-related genes zeaxanthin cleavage dioxygenase $($ CaZCD), lycopene $\beta$-cyclase (CaLYCB), phytoene desaturase (CaPDS). The expression of these genes was normalized to CaUbi3. $\mathrm{N}$ and $\mathrm{L}$ represent normal conditions and low temperature, respectively. The experiment was conducted with three biological replicates, and each replicate contained three pepper seedlings. Means \pm SDs, different lowercase letters mark significant differences at $P<0.05$. 


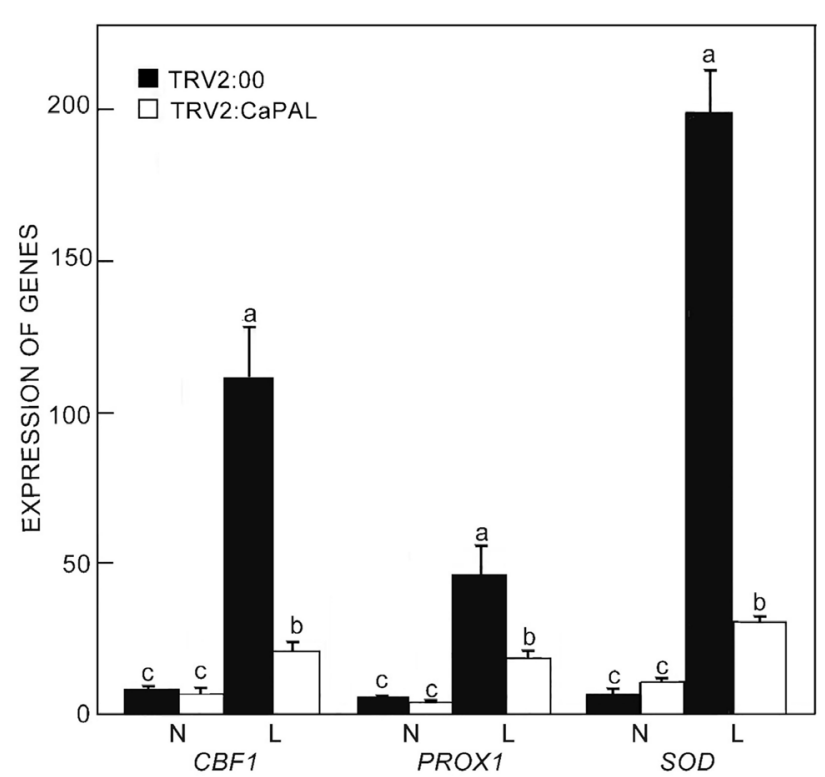

Fig. 6. Changes in antioxidant-related gene (C-repeat binding factor 1 - CBF1, proline oxidoreductase 1 - PROX1, and superoxide dismutase - SOD) expressions of CaPAL-silenced peppers TRV2-CaPAL and negative control plants TRV2:00 exposed to low temperature $\left(4{ }^{\circ} \mathrm{C}\right.$ for $\left.16 \mathrm{~h}\right)$. Gene expressions were normalized to $\mathrm{CaUbi3}$ expression. The experiment was conducted with three biological replicates, and each replicate contained three pepper seedlings. Means \pm SDs, different lowercase letters mark significant differences at $P<0.05$.

growth and development (Zhao et al. 2016). Plants possess an extensive spectrum of antioxidants and antioxidative enzymes that allow survival under stress conditions, ensure optimal cellular ROS content to avoid damage, and allow signaling to proceed. This system is highly dynamic and involves both generation and scavenging ROS to retain balanced levels in plant cells (Mittler et al. 2004). Here, under low temperature conditions, enhanced activities of antioxidant enzymes in both the negative control and the CaPAL-silenced pepper plants may have been induced to scavenge a higher ROS production. This is also supported by the higher expression of antioxidant genes under low temperature (Fig. 6).

In order to further confirm the contribution of CaPAL to the pepper plant tolerance to low temperature, photosynthetic parameters were investigated under low temperature conditions. It is known that anthocyanins can improve photosynthetic capacity under low temperature. At low temperatures, plant can maintain growth and development by accumulating anthocyanins (Zhou et al. 2017). Anthocyanins are located in the outer palisade cells, protecting the photosynthetic tissues from excessive radiation. An increase of anthocyanin content is positively correlated with photosynthesis capacity (Devacht et al. 2009). Anthocyanins can protect the photosynthesis apparatus in stressful environments most likely through absorbing visible radiation and shielding UV radiation in plants exposed to low temperature (Chen et al. 2007). On the contrary, anthocyanin deficiency in the CaPALsilenced peppers inhibited photosynthetic rate under low temperature probably due to loss of photoprotection. These data suggest the positive role of anthocyanins in photosynthetic capacity.

\section{Conclusions}

We found that inhibiting CaPAL expression in the pepper by VIGS disrupted biosynthesis of anthocyanins and caused abnormal development. The CaPAL-silenced peppers were more sensitive to low temperature showed a reduced ability to remove ROS, and had a lower photosynthetic capacity. At the molecular level, low transcriptions of genes involved in pigment biosynthesis and antioxidant system support the result. Our work provides an insight into the role of PAL in anthocyanin biosynthesis and tolerance to low temperature stress.

\section{References}

Aza-González, C., Herrera-Isidrón, L., Núñez-Palenius, H.G., Martínez De La Vega, O., Ochoa-Alejo, N.: Anthocyanin accumulation and expression analysis of biosynthesis-related genes during chili pepper fruit development. - Biol. Plant. 57: 49-55, 2013.

Chen, J., Chen, Q., Cheng, Z.: Protective effect of anthocyanin on photosynthetic apparatus of tomato ( $L$. esculentum Mill.) seedling leaves exposed to low temperature and low irradiance. - Sci. Agr. sin. 40: 788-793, 2007. [In Chinese]

Cheng, G.X., Li, R.J., Wang, M., Huang, L.J., Khan, A., Ali, M., Gong, Z.H.: Variation in leaf color and combine effect of pigments on physiology and resistance to whitefly of pepper ( Capsicum annuum L.). - Scientia Hort. 229: 215-225, 2018.

Cheng, G.W., Breen, P.J.: Activity of phenylalanine ammonialyase (PAL) and concentrations of anthocyanins and phenolics in developing strawberry fruit. - J. amer. Soc. hort. Scl. 116: 865-869, 1991.

Christie, P.J., Alfenito, M.R., Walbot, V.: Impact of lowtemperature stress on general phenylpropanoid and anthocyanin pathways: enhancement of transcript abundance and anthocyanin pigmentation in maize seedlings. - Planta 194: 541-549, 1994.

Christopoulos, M.V., Tsantili, E.: Participation of phenylalanine ammonia-lyase (PAL) in increased phenolic compounds in fresh cold stressed walnut (Juglans regia L.) kernels. Postharvest Biol. Technol. 104: 17-25, 2015.

Conde, A., Chaves, M.M., Gerós, H.: Membrane transport, sensing and signaling in plant adaptation to environmental stress. - Plant Cell Physiol. 52: 1583-1602, 2011.

Cunningham, F.X., Gantt, E.: Genes and enzymes of carotenoid biosynthesis in palnts. - Annu. Rev. Plant Physiol. Plant mol.

Biol. 49: 557-583, 1998

Devacht, S., Lootens, P., Baert, J.V., Bockstaele, E.V., RoldanRuiz, I.: Influence of anthocyanin on the photosynthetic performance of industrial chicory under cold stress conditions. - Commun. Agr. appl. Biol. Sci. 74: 121-126, 2009.

Dionisio-Sese, M.L., Tobita, S.: Antioxidant responses of rice seedlings to salinity stress. - Plant Sci. 135: 1-9, 1998.

Fantini, E., Giuliano, G.: Virus-induced gene silencing as a tool to study tomato fruit biochemistry. - Methods mol. Biol. 1363: 65-78, 2016.

Feng, H., Li, Y., Liu, Z., Liu, J.: Mapping of or, a gene conferring orange color on the inner leaf of the Chinese cabbage (Brassica 
rapa L. ssp. pekinensis). - Mol. Breed. 29: 235-244, 2012.

Filiz, A., Bürün, B., Nurettin Ş.: Fungal contaminants observed during micropropagation of Lilium candidum $\mathrm{L}$. and the effect of chemotherapeutic substances applied after sterilization. Afr. J. Biotechnol. 9: 991-995, 2010.

Guo, W.L., Chen, R.G., Gong, Z.H., Yin, Y.X., Ahmed, S.S.: Exogenous abscisic acid increases antioxidant enzymes and related gene expression in pepper ( Capsicum annuum ) leaves subjected to chilling stress. - Genet. mol. Res. 11: 4063-4080, 2012.

Huang, J., Gu, M., Lai, Z., Fan, B., Shi, K., Zhou, Y.H., Yu, J.Q., Chen, Z.: Functional analysis of the Arabidopsis PAL gene family in plant growth, development, and response to environmental stress. - Plant Physiol. 153: 1526-1538, 2010.

Jenshi Roobha, J., Saravanakumar, M. Aravindhan, K.M., Devi, P.S.: The effect of light, temperature, $\mathrm{pH}$ on stability of anthocyanin pigments in Musa acuminata bract. - Res. Plant Biol. 1: 5-12, 2011.

Jeong, R.D., Hwang, S.H., Kang, S.H., Choi, H.S., Park, J.W., Kim, K.H.: Virus-induced gene silencing as tool for functional genomics in a Glycine max. - Plant Pathol. J. 21: 158-163, 2005.

Jupin, I.: A protocol for VIGS in Arabidopsis thaliana using a one-step TYMV-derived vector. - Methods mol. Biol. 975: 197-210, 2013.

Kim, D.S., Hwang, B.K.: An important role of the pepper phenylalanine ammonia-lyase gene $(P A L 1)$ in salicylic aciddependent signalling of the defence response to microbial pathogens. - J. exp. Bot. 65: 2295-2306, 2014.

Kovačević, D.B., Putnik, P., Dragović-Uzelac, V., Vahcić N., Babojelić, M.S., Levaj B.: Influences of organically and conventionally grown strawberry cultivars on anthocyanins content and color in purees and low-sugar jams. - Food Chem. 181: 94-100, 2015.

Landi, M., Tattini, M., Gould, K.S.: Multiple functional roles of anthocyanins in plant-environment interactions. - Environ. exp. Bot. 119: 4-17, 2015.

Leyva, A., Jarillo, J.A., Salinas, J., Martinez-Zapater, J.M.: Low temperature IiSynthase?? mRNAs of Arabidopsis thaliana in a light-dependent manner. - Plant Physiol. 108: 39-46, 1995.

Mauch-Mani, B., Slusarenko, A.J.: Production of salicylic acid precursors is a major function of phenylalanine ammonialyase in the resistance of Arabidopsis to Peronospora parasitica. - Plant Cell 8: 203-212, 1996.

Mittler, R., Vanderauwera, S., Gollery, M., Van Breusegem F.: Reactive oxygen gene network of plants. - Trends Plant Sci. 9: 490-498, 2004.

Nakano, Y., Asada, K.: Purification of ascorbate peroxidase in spinach chloroplasts; its inactivation in ascorbate-depleted medium and reactivation by monodehydroascorbate radical. - Plant Cell Physiol. 28: 131-140, 1987.

Olsen, K.M., Lea, U.S., Slimestad, R., Verheul, M., Lillo, C.: Differential expression of four Arabidopsis PAL genes; $P A L 1$ and PAL2 have functional specialization in abiotic environmental-triggered flavonoid synthesis. - J. Plant Physiol. 165: 1491-1499, 2008.

Oren-Shamir, M.: Does anthocyanin degradation play a significant role in determining pigment concentration in plants? - Plant Sci. 177: 310-316, 2009.

Pimenta, S., Menezes, D., Neder, D.G., Melo. R.A.: Organic production systems. - Horticult. Bras. 34: 168-174, 2016.

Pombo, M.A., Martínez, G.A., Civello, P.M.: Cloning of FaPAL6 gene from strawberry fruit and characterization of its expression and enzymatic activity in two cultivars with different anthocyanin accumulation. - Plant Sci. 181: 111-118,
2011.

Porra, R.J., Thompson, W., Kriedemann, P.E.: Determination of accurate extinction coefficients and simultaneous-equations for assaying chlorophyll $a$ and chlorophyll $b$ extracted with 4 different solvents - verification of the concentration of chlorophyll standards by atomic-absorption spectroscopy. BBA - Biomembranes 975: 384-394, 1989.

Purkayastha, A., Mathur, S., Verma, V., Sharma, S., Dasgupta, I.: Virus-induced gene silencing in rice using a vector derived from a DNA virus. - Planta 232: 1531-1540, 2010.

Ramegowda, V., Mysore, K.S., Senthil-Kumar, M. Virus-induced gene silencing is a versatile tool for unraveling the functional relevance of multiple abiotic-stress-responsive genes in crop plants. - Front. Plant Sci. 5: 323. 2014.

Rohde, A.: Molecular phenotyping of the pall and pal2 mutants of Arabidopsis thaliana reveals far-reaching consequences on phenylpropanoid, amino acid, and carbohydrate metabolism. - Plant Cell 16: 2749-2771, 2004.

Schulz, E., Tohge, T., Zuther, E., Fernie, A.R., Hincha, D.K.: Natural variation in flavonol and anthocyanin metabolism during cold acclimation in Arabidopsis thaliana accessions. - Plant Cell Eniron. 38:1658-1672, 2015.

Tavakol, E. Virus-induced gene silencing (VIGS) in Aegilops tauschii and its use in functional analysis of AetDREB2. Mol. Biotechnol. 60: 41-48, 2018.

Tonnessen, B.W., Manosalva, P., Lang, J.M., Baraoidan, M., Bordeos, A., Mauleon, R., Oard, J., Hulbert, S., Leung, H., Leach, J.E.: Rice phenylalanine ammonia-lyase gene OSPAL4 is associated with broad spectrum disease resistance. - Plant mol. Biol. 87: 273-286, 2014.

Ubi B.: Molecular mechanisms underlying anthocyanin biosynthesis: a useful tool for the metabolic engineering of the flavonoid pathway genes for novel products. - J. Food Agr. Environ. 5: 83-87, 2007.

Van Tunen, A.J., Mol, J.N.M.: Control of Flavonoids Synthesis and Manipulation of Flower Color. - Springer, Dordrecht 1991.

Wang, H., Arakawa, O., Motomura, Y.: Influence of maturity and bagging on the relationship between anthocyanin accumulation and phenylalanine ammonia-lyase (PAL) activity in 'Jonathan' apples. - Postharvest Biol. Technol. 19: 123-128, 2000.

Zhai, Y., Wang, H., Liang, M., Lu, M.: Both silencing- and over-expression of pepper $C a A T G 8 c$ gene compromise plant tolerance to heat and salt stress. - Environ. exp. Bot. 141: 10$18,2017$.

Zhao, X., Chen, T., Feng, B., Zhang, C., Peng, S., Zhang, X., Fu, G., Tao, L.: Non-photochemical quenching plays a key role in light acclimation of rice plants differing in leaf color. - Front. Plant Sci. 7: 1968, 2016.

Zhou, J.X., Wang, S.Q.: Activity of phenylalanine ammonia lyase and the changes of some nitrogenous compounds in oligophenol cotton. - Plant Physiol. Commun. ??: 39-40, 1990.

Zhou, L.J., Li, Y.Y., Zhang, R.F., Zhang, C.L., Xie, X.B., Zhao, C., Hao, Y.J.: The small ubiquitin-like modifier E3 ligase MdSIZ1 promotes anthocyanin accumulation by sumoylating MdMYB1 under low-temperature conditions in apple. - Plant Cell Environ. 40: 2068-2080, 2017.

Zhu, Q., Xie, X., Lin, H., Sui, S., Shen, R., Yang, Z., Lu, K., Li, M., Liu, Y.G.: Isolation and functional characterization of a phenylalanine ammonia-lyase gene $(S S P A L 1)$ from coleus (Solenostemon scutellarioides (L.) Codd). - Molecules 20: 16833-16851, 2015. 